Pacific Journal of Mathematics

ON OPERATORS WHOSE SPECTRUM LIES ON A CIRCLE OR 


\section{ON OPERATORS WHOSE SPECTRUM LIES ON A CIRCLE OR A LINE}

\section{HERBERT KAMOWITZ}

The purpose of this note is to prove the following theorem.

Theorem. Let $N$ be a bounded linear operator on Hilbert space $H$ satisfying

$$
\|N T-T N\|=\left\|N^{*} T-T N^{*}\right\|
$$

for all bounded linear operators $T$. Then $N$ is (obviously) normal and the spectrum of $N$ lies on a circle or straight line.

Here $N^{*}$ denotes the adjoint of the operator $\mathbf{N}$.

It is clear that if $S$ is a unitary or self-adjoint operator and $\alpha$ and $\beta$ are complex numbers, then $N=\alpha I+\beta S$ satisfies (1). The theorem asserts that the converse is also true.

After noting that in dimensions two and three the theorem is trivially true, we proceed to the first of two parts of the proof.

I. Dimension four. Let $H$ be four dimensional Euclidean space. Since a normal operator $N$ is unitarily equivalent to a diagonal matrix it is no restriction to assume that $N$ has the form

$$
\left(\begin{array}{llll}
\lambda_{1} & 0 & 0 & 0 \\
0 & \lambda_{2} & 0 & 0 \\
0 & 0 & \lambda_{3} & 0 \\
0 & 0 & 0 & \lambda_{4}
\end{array}\right)
$$

where the $\lambda_{i}$ 's are the eigenvalues of $N$. Consider the matrix

$$
T=\left(\begin{array}{llll}
0 & 0 & 1 & 1 \\
0 & 0 & 1 & i \\
0 & 0 & 0 & 0 \\
0 & 0 & 0 & 0
\end{array}\right)
$$

Then

$$
N T-T N=\left(\begin{array}{cccc}
0 & 0 & \left(\lambda_{1}-\lambda_{3}\right) & \left(\lambda_{1}-\lambda_{4}\right) \\
0 & 0 & \left(\lambda_{2}-\lambda_{3}\right) & i\left(\lambda_{2}-\lambda_{4}\right) \\
0 & 0 & 0 & 0 \\
0 & 0 & 0 & 0
\end{array}\right)
$$

and $\|N T-T N\|^{2}$ is the largest eigenvalue of the matrix 


$$
A=\left(\begin{array}{cccc}
\left|\lambda_{1}-\lambda_{3}\right|^{2}+\left|\lambda_{1}-\lambda_{4}\right|^{2} & \left(\lambda_{1}-\lambda_{3}\right) \overline{\left(\lambda_{2}-\lambda_{3}\right)} & 0 & 0 \\
\overline{\left(\overline{\left.\lambda_{1}-\lambda_{3}\right)}\left(\lambda_{2}-\lambda_{3}\right)\right.} & -i\left(\lambda_{1}-\lambda_{4}\right)\left(\overline{\left.\lambda_{2}-\lambda_{4}\right)}\right. & & \\
+i \overline{\left(\lambda_{1}-\lambda_{4}\right)}\left(\lambda_{2}-\lambda_{4}\right) & \left|\lambda_{2}-\lambda_{3}\right|^{2}+\left|\lambda_{2}-\lambda_{4}\right|^{2} & 0 & 0 \\
0 & 0 & 0 & 0 \\
0 & 0 & 0 & 0
\end{array}\right) .
$$

On the other hand, $\left\|N^{*} T-T N^{*}\right\|^{2}$ is the largest eigenvalue of a matrix $B$ which has the same form as $A$ but with $\lambda_{i}$ replaced by $\bar{\lambda}_{i}$, $i=1,2,3,4$.

Let $P(X)$ and $P^{*}(X)$ be the characteristic polynomials of $A$ and $B$, respectively. Then from the form of the matrices $A$ and $B$ one sees that the polynomials $P(X)$ and $P^{*}(X)$ differ only in the coefficient of $X^{2}$. Therefore $\|N T-T N\|=\left\|N^{*} T-T N^{*}\right\|$ if, and only if, $P(X)$ and $P^{*}(X)$ are equal and a routine computation shows that this holds if, and only if,

$$
\begin{aligned}
& {\left[\left(\overline{\lambda_{1}-\lambda_{3}}\right)\left(\lambda_{2}-\lambda_{3}\right)+\right.}\left.i\left(\overline{\lambda_{1}-\lambda_{4}}\right)\left(\lambda_{2}-\lambda_{4}\right)\right] \\
& \times\left[\left(\lambda_{1}-\lambda_{3}\right)\left(\overline{\lambda_{2}-\lambda_{3}}\right)-i\left(\lambda_{1}-\lambda_{4}\right)\left(\overline{\lambda_{2}-\lambda_{4}}\right)\right] \\
&=\left[\left(\lambda_{1}-\lambda_{3}\right)\left(\overline{\lambda_{2}-\lambda_{3}}\right)+i\left(\lambda_{1}-\lambda_{4}\right)\left(\overline{\lambda_{2}-\lambda_{4}}\right)\right] \\
& \times\left[\left(\overline{\lambda_{1}-\lambda_{3}}\right)\left(\overline{\lambda_{2}}-\lambda_{3}\right)-i\left(\overline{\lambda_{1}-\lambda_{4}}\right)\left(\lambda_{2}-\lambda_{4}\right)\right]
\end{aligned}
$$

which reduces to the condition that $\left(\overline{\lambda_{1}-\lambda_{4}}\right)\left(\overline{\lambda_{2}-\lambda_{3}}\right)\left(\lambda_{1}-\lambda_{3}\right)\left(\lambda_{2}-\lambda_{4}\right)$ be real. The latter holds if, and only if, $\lambda_{1}, \lambda_{2}, \lambda_{3}, \lambda_{4}$ lie on a line or circle, which proves the theorem in dimension four.

Let $\left(a_{1}, a_{2}, a_{3}, a_{2}\right)$ be a vector in four dimensional $H$. Since

$$
\begin{aligned}
& (N T-T N)\left(a_{1}, a_{2}, a_{3}, a_{4}\right) \\
& \quad=\left(\left(\lambda_{1}-\lambda_{3}\right) a_{3}+\left(\lambda_{1}-\lambda_{4}\right) a_{4},\left(\lambda_{2}-\lambda_{3}\right) a_{3}+i\left(\lambda_{2}-\lambda_{4}\right) a_{4}, 0,0\right),
\end{aligned}
$$

we remark that we have just shown that

(2) $\sup _{\Sigma\left|a_{i}\right|^{2}=1}\left[\left|\left(\lambda_{1}-\lambda_{3}\right) a_{3}+\left(\lambda_{1}-\lambda_{4}\right) a_{4}\right|^{2}+\left|\left(\lambda_{2}-\lambda_{3}\right) a_{3}+i\left(\lambda_{2}-\lambda_{4}\right) a_{4}\right|^{2}\right]$ is the largest root of $P(X)=0$, and also that

$$
\sup _{\Sigma\left|a_{i}\right|^{2}=1}\left[\left|\left(\overline{\lambda_{1}-\lambda_{3}}\right) a_{3}+\left(\overline{\lambda_{1}-\lambda_{3}}\right) a_{4}\right|^{2}+\left|\left(\overline{\lambda_{2}-\lambda_{3}}\right) a_{3}+i\left(\overline{\lambda_{2}-\lambda_{4}}\right) a_{4}\right|^{2}\right]
$$

is the largest root of $P^{*}(X)=0$.

II. Higher dimensions. Now let $H$ be a finite or infinite dimensional Hilbert space of dimension $>4$. Let $N$ be a normal operator satisfying $\|N T-T N\|=\left\|N^{*} T-T N^{*}\right\|$ for all $T$. Suppose $N$ has spectrum $S$ and spectral representation $N=\int_{S} \lambda d E_{\lambda}$. Let 
$\lambda_{1}, \lambda_{2}, \lambda_{3}, \lambda_{4}$ be distinct points of $S$. (If $S$ has no more than three points then the theorem is trivially true.) Choose an $\varepsilon>0$ but smaller than the minimum of the distances $\left|\lambda_{i}-\lambda_{j}\right|, i \neq j$; let $S_{k}=$ $S \cap\left\{\lambda|| \lambda-\lambda_{k} \mid<\varepsilon\right\}, k=1,2,3,4$. Let $E_{k}=\int_{S_{k}} d E_{\lambda}$ and choose $y \in H$ with $\left\|E_{k} y\right\|=1$ for $k=1,2,3,4$ and let $x_{k}=E_{k} y$. Then $\left(x_{i}, x_{j}\right)=0$ if $i \neq j$ and each $x_{k}$ is an approximate eigenvector in the sense that

$$
\begin{aligned}
\left\|N x_{k}-\lambda_{k} x_{k}\right\| & =\left\|\int_{S_{k}} \lambda d E_{\lambda} x_{k}-\lambda_{k} \int_{S_{k}} d E_{\lambda} x_{k}\right\| \\
& \leqq \max _{\lambda \in S_{k}}\left|\lambda-\lambda_{k}\right|<\varepsilon .
\end{aligned}
$$

We can therefore write $N x_{k}=\lambda_{k} x_{k}+\varepsilon u_{k}$ where $\left\|u_{k}\right\| \leqq 1$. Similarly $N^{*} x_{k}=\bar{\lambda}_{k} x_{k}+\varepsilon u_{k}^{\prime},\left\|u_{k}^{\prime}\right\| \leqq 1$.

Define an operator $T$ on $H$ by

$$
T x=\left[\left(E_{3} x, x_{3}\right)+\left(E_{4} x, x_{4}\right)\right] x_{1}+\left[\left(E_{3} x, x_{3}\right)+i\left(E_{4} x, x_{4}\right)\right] x_{2} .
$$

Now for $x \in H$,

$$
\begin{aligned}
N T x= & {\left[\left(E_{3} x, x_{3}\right)+\left(E_{4} x, x_{4}\right)\right] \lambda_{1} x_{1} } \\
& +\left[\left(E_{3} x, x_{3}\right)+i\left(E_{4} x, x_{4}\right)\right] \lambda_{2} x_{2}+\delta_{1}(x),
\end{aligned}
$$

where $\left\|\delta_{1}(x)\right\| \leqq 4 \varepsilon\|x\|$. Also

$$
\begin{aligned}
T(N x)= & {\left[\left(E_{3} N x, x_{3}\right)+\left(E_{4} N x, x_{4}\right)\right] x_{1} } \\
& +\left[\left(E_{3} N x, x_{3}\right)+i\left(E_{4} N x, x_{4}\right)\right] x_{2} .
\end{aligned}
$$

Since $N E_{k}=E_{k} N, k=1,2,3,4$, it follows that

$$
\begin{aligned}
T N x= & {\left[\left(E_{3} x, \bar{\lambda}_{3} x_{3}\right)+\left(E_{4} x, \bar{\lambda}_{4} x_{4}\right)\right] x_{1} } \\
& +\left[\left(E_{3} x, \bar{\lambda}_{3} x_{3}\right)+i\left(E_{4} x, \bar{\lambda}_{4} x_{4}\right)\right]+\delta_{2}(x),
\end{aligned}
$$

where $\left\|\delta_{2}(x)\right\| \leqq 4 \varepsilon\|x\|$.

Thus

$$
\begin{aligned}
& \|(N T-T N) x\| \\
& =\|\left[\left(\lambda_{1}-\lambda_{3}\right)\left(E_{3} x, x_{3}\right)+\left(\lambda_{1}-\lambda_{4}\right)\left(E_{4} x, x_{4}\right)\right] x_{1} \\
& \quad+\left[\left(\lambda_{2}-\lambda_{3}\right)\left(E_{3} x, x_{3}\right)+i\left(\lambda_{2}-\lambda_{\sharp}\right)\left(E_{4} x, x_{4}\right)\right] x_{2}+\gamma_{1}(x) \|,
\end{aligned}
$$

where $\left\|\gamma_{1}(x)\right\| \leqq 8 \varepsilon\|x\|$.

Similarly

$$
\begin{aligned}
& \left\|\left(N^{*} T-T N^{*}\right) x\right\| \\
& =\|\left[\left(\overline{\lambda_{1}-\lambda_{3}}\right)\left(E_{3} x, x_{3}\right)+\left(\overline{\lambda_{1}-\lambda_{4}}\right)\left(E_{4} x, x_{4}\right)\right] x_{1} \\
& \quad+\left[\left(\overline{\lambda_{2}-\lambda_{3}}\right)\left(E_{3} x, x_{3}\right)+i\left(\overline{\lambda_{2}-\lambda_{4}}\right)\left(E_{4} x, x_{4}\right)\right] x_{2}+\gamma_{2}(x) \|,
\end{aligned}
$$

where $\left\|\gamma_{2}(x)\right\| \leqq 8 \varepsilon\|x\|$. 
Since $x_{1}$ and $x_{2}$ are orthogonal,

$$
\begin{aligned}
& \|N T-T N\|^{2} \\
& =\sup _{\|x\|=1}\left[\left|\left(\lambda_{1}-\lambda_{3}\right)\left(E_{3} x, x_{3}\right)+\left(\lambda_{1}-\lambda_{4}\right)\left(E_{i} x, x_{4}\right)\right|^{2}\right. \\
& \left.+\left|\left(\lambda_{2}-\lambda_{3}\right)\left(E_{3} x, x_{3}\right)+i\left(\lambda_{2}-\lambda_{4}\right)\left(E_{4} x, x_{4}\right)\right|^{2}\right]+\delta
\end{aligned}
$$

and

$$
\begin{aligned}
\| N^{*} T & -T N^{*} \|^{2} \\
= & \sup _{\|\| x \|=1}\left[\left|\left(\overline{\lambda_{1}-\lambda_{3}}\right)\left(E_{3} x, x_{3}\right)+\left(\overline{\lambda_{1}-\lambda_{4}}\right)\left(E_{4} x, x_{4}\right)\right|^{2}\right. \\
& \left.+\left|\left(\overline{\lambda_{2}-\lambda_{3}}\right)\left(E_{3} x, x_{3}\right)+i\left(\overline{\lambda_{2}-\lambda_{4}}\right)\left(E_{4} x, x_{4}\right)\right|^{2}\right]+\delta^{\prime},
\end{aligned}
$$

where $\delta, \delta^{\prime} \leqq 8 \varepsilon$.

Thus if $\|N T-T N\|=\left\|N^{*} T-T N^{*}\right\|$, we obtain by (2) and (3) that the largest zeros of $P(X)$ and $P^{*}(X)$ differ by at most $16 \varepsilon$. Therefore all the coefficients of $P(X)$ and $P^{*}(X)$ are close to each other and in particular the imaginary part of

$$
\left(\overline{\lambda_{1}-\lambda_{1}}\right)\left(\overline{\lambda_{2}-\lambda_{3}}\right)\left(\lambda_{1}-\lambda_{3}\right)\left(\lambda_{2}-\lambda_{4}\right)
$$

is bounded by a function of $\varepsilon$ which goes to zero with $\varepsilon$. Letting $\varepsilon \rightarrow 0$ we again find that $\left(\overline{\lambda_{1}-\lambda_{4}}\right)\left(\overline{\lambda_{2}-\lambda_{3}}\right)\left(\lambda_{1}-\lambda_{3}\right)\left(\lambda_{2}-\lambda_{4}\right)$ must be real and so $\lambda_{1}, \lambda_{2}, \lambda_{3}$ and $\lambda_{4}$ lie on a line or a circle. Since the $\lambda_{i}$ 's were arbitrary, the proof is concluded.

Received April 27, 1965.

Avco Corporation

Wilmington, Massachusetts

University of Massachusetts

Boston, Massachusetts 


\section{PACIFIC JOURNAL OF MATHEMATICS}

H. SAMELSON

Stanford University

Stanford, California

J. P. JaNs

University of Washington

Seattle, Washington 98105

\section{EDITORS}

J. DugundJI

University of Southern California Los Angeles, California 90007

RICHARD ARENS

University of California

Los Angeles, California 90024

\section{ASSOCIATE EDITORS}

E. F. BECKENBACH

B. H. NEUMANN

F. WOLF

K. YosidA

\section{SUPPORTING INSTITUTIONS}

UNIVERSITY OF BRITISH COLUMBIA

CALIFORNIA INSTITUTE OF TECHNOLOGY

UNIVERSITY OF CALIFORNIA

MONTANA STATE UNIVERSITY

UNIVERSITY OF NEVADA

NEW MEXICO STATE UNIVERSITY

OREGON STATE UNIVERSITY

UNIVERSITY OF OREGON

OSAKA UNIVERSITY

UNIVERSITY OF SOUTHERN CALIFORNIA
STANFORD UNIVERSITY

UNIVERSITY OF TOKYO

UNIVERSITY OF UTAH

WASHINGTON STATE UNIVERSITY

UNIVERSITY OF WASHINGTON

AMERICAN MATHEMATICAL SOCIETY CHEVRON RESEARCH CORPORATION TRW SYSTEMS

NAVAL ORDNANCE TEST STATION 


\section{Pacific Journal of Mathematics}

\section{Vol. 20, No. $1 \quad$ September, 1967}

Leonard Daniel Baumert, Extreme copositive quadratic forms. II ........ 1

Edward Lee Bethel, A note on continuous collections of disjoint

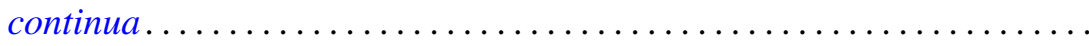

Delmar L. Boyer and Adolf G. Mader, A representation theorem for abelian groups with no elements of infinite p-height ...................

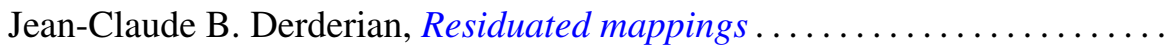

Burton I. Fein, Representations of direct products of finite groups ......... 45

John Brady Garnett, A topological characterization of Gleason parts.......

Herbert Meyer Kamowitz, On operators whose spectrum lies on a circle or

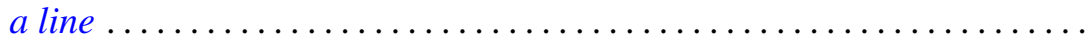

Ignacy I. Kotlarski, On characterizing the gamma and the normal distribution ........................................

Yu-Lee Lee, Topologies with the same class of homeomorphisms ..........

Moshe Mangad, Asymptotic expansions of Fourier transforms and discrete polyharmonic Green's functions ...........................

Jürg Thomas Marti, On integro-differential equations in Banach spaces ....

Walter Philipp, Some metrical theorems in number theory.............. 109

Maxwell Alexander Rosenlicht, Another proof of a theorem on rational

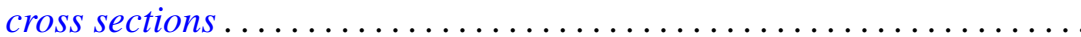

Kenneth Allen Ross and Karl Robert Stromberg, Jessen's theorem on Riemann sums for locally compact groups

Stephen Simons, A theorem on lattice ordered groups, results of Ptak, Namioka and Banach, and a front-ended proof of Lebesgue's theorem...

Morton Lincoln Slater, On the equation $\varphi(x)=\int_{x} x+1 K(\xi) f[\varphi(\xi)] d \xi \ldots$ 155 Arthur William John Stoddart, Existence of optimal controls .. 167 Burnett Roland Toskey, A system of canonical forms for rings on a direct sum of two infinite cyclic groups ....................

Jerry Eugene Vaughan, A modification of Morita's characterization of dimension 